\title{
Preferencias de investigadores y prácticas institucionales/disciplinares en la difusión y socialización de los resultados de investigación
}

Sandra Miguel'1, Claudia M. González¹ y Edgardo Ortiz-Jaureguizar²

\begin{abstract}
${ }^{1}$ Instituto de Investigaciones en Humanidades y Ciencias Sociales (IdIHCS) (UNLP-CONICET), Facultad de Humanidades y Ciencias de la Educación, Universidad Nacional de La Plata, Argentina / smiguel@fahce.unlp.edu.ar | https://orcid.org/ooooooo1-9384-6838 / cgonzalez@fahce.unlp.edu.ar | https://orcid.org/oooo-ooo1-9145-3539

${ }^{2}$ Laboratorio de Sistemática y Biología Evolutiva (LASBE), Facultad de Ciencias Naturales y Museo, Universidad Nacional de La Plata. Argentina / CONICET / eortiz@fcnym.unlp.edu.ar | https://orcid.org/oooo-00o2-4333-1606
\end{abstract}

\section{Resumen}

El objetivo de este trabajo es realizar un aporte al conocimiento de las prácticas y preferencias de los docentes-investigadores de instituciones universitarias al momento de difundir y socializar sus publicaciones en repositorios institucionales de acceso abierto y en redes sociales académicas, partiendo de la premisa de que dicho fenómeno está influenciado tanto por la disciplina, como por la pertenencia institucional. Con una aproximación bibliométrica-cienciométrica se estudia la presencia de la comunidad académica y el volumen y cobertura temporal de su producción depositada en repositorios institucionales (RIS) y en la red social académica ResearchGate (RG). Se estudia el caso de docentes-investigadores de tres facultades de una universidad pública argentina, caracterizando a la población según género, grupo etario, grado académico y categoría formalizada dentro del sistema de investigación. Se concluye que hay mayor presencia de la comunidad académica en RI que en RG, aunque el promedio de documentos por docente investigador es más elevado en RG. Las comunidades de las facultades de Ciencias Exactas y Ciencias Naturales tienen alta presencia en RG a diferencia de las de Humanidades y Ciencias Sociales. Hay más mujeres que hombres en las distintas plataformas, a la inversa de lo que ocurre con el promedio de documentos por docente investigador, que también es más elevado cuanto mayor es la edad, la categoría de investigador, y el grado académico alcanzado. La cobertura temporal y la actualidad de la producción es mayor en RG que en RI, además de existir una relación entre la antigüedad de los documentos y el grupo etario de la población.

\begin{abstract}
Preferences of researchers and institutional/disciplinary practices in the dissemination and socialisation of research results. The objective of this work is to contribute to the knowledge of the practices and preferences of university researchers when they have to disseminate and socialize their publications in open access institutional
\end{abstract}

\section{Palabras clave}

Repositorios institucionales Redes sociales académicas ResearchGate Bibliometría Altmetría

\section{Keywords}

Institutional repositories Academic social networks ResearchGate Bibliometrics Altmetrics 
repositories and academic social networks. This study is based on the premise that this phenomenon is influenced both by discipline and by institutional belonging. With a bibliometric-scientometric approach, the presence of the academic community and the volume and temporal coverage of its production deposited in institutional repositories (RIS) and the academic social network ResearchGate (RG) are studied. The case of university researchers from three faculties of an Argentine public university is studied, characterizing the population according to gender, age group, academic degree, and category formalized within the research system. It is concluded that there is a higher presence of the academic community in the repositories than in the social network, although the average of documents per university researcher is higher in the latter. The community of the faculties of Exact Sciences and Natural Sciences have a high presence in RG, unlike those in Humanities and Social Sciences. There are more women than men in the different platforms, in contrast to what happens with the average number of documents per university researcher, which is also higher the higher the age, the researcher category, and the academic degree achieved. Temporary coverage and current production are higher in RG than in the RIS, in addition to a relationship between the age of the documents and the age group of the population.

Artículo recibido: 5-12-2017. Aceptado: 9-04-2018

\section{Introducción}

La comunicación científica es un aspecto inherente a la naturaleza misma de la ciencia. Abarca un amplio espectro de actividades relacionadas con la producción, diseminación y uso de la información, y comprende los diferentes canales, formales e informales, que utilizan los investigadores en cualquier campo para dar a conocer los resultados de sus investigaciones (Garvey y Griffith, 1972; Borgman, 1989). Si bien los estudios sobre la comunicación científica vienen de larga data, han cobrado especial relevancia en los últimos años en los que el uso de las tecnologías de la web 2.0 por parte de la comunidad científica, el desarrollo del movimiento de acceso abierto a las publicaciones y el surgimiento de nuevos indicadores para estimar el impacto de la investigación en la web social, están transformando la estructura general del sistema de comunicación y evaluación de la ciencia (Hurd, 2000; Liu, 2003; Björk, 2007; Russell Barnard, 2007; Ortega, 2015).

A los canales tradicionales de publicación y difusión de los resultados de la investigación se suman en la actualidad nuevas plataformas basadas en el uso de tecnologías participativas de la web 2.0, que amplían los espacios de comunicación e interacción entre los científicos. A las redes sociales de perfil público, como Facebook y Twitter, se agregan otras específicas de carácter académico, como ResearchGate y Academia.edu, las que a partir de su creación en 2008 se han ido convirtiendo paulatinamente en las más populares de la web social académica (Echavarría Ramírez, 2010; Santana Arroyo, 2010; Alba-Ruiz et al., 2011). El número de usuarios y el volumen de documentos que registran evidencian el grado de aceptación que tienen en la comunidad científica a nivel mundial. Según estadísticas de las propias fuentes, a marzo de 2017 ResearchGate reunía 12 millones de miembros y más de 100 millones de publicaciones, y Academia.edu 49 millones de investigadores y cerca de 8 millones de documentos. Su crecimiento en los últimos años $-320 \%$ para la primera y $43 \%$ para la segunda-, revela además que el número de investigadores con presencia en ellas va en aumento (Ortega, 2015).

En forma paralela al desarrollo y crecimiento de la web social académica, el surgimiento del movimiento de acceso abierto también produjo cambios en el proceso de la comunicación científica. El acceso abierto (AA) propone la disponibilidad gratuita 
en la Internet pública de la literatura científica, de modo que cualquier usuario pueda leer, descargar, copiar, distribuir, imprimir, buscar o añadir un enlace al texto completo de los artículos, recuperarlos para su indización, incorporarlos como datos en un software, o utilizarlos para cualquier otro propósito, sin barreras financieras, legales o técnicas, aparte de las que son inherentes al acceso mismo a Internet (BOAI, 2002, 2015). La publicación en revistas AA (vía dorada) o el autoarchivo en repositorios digitales, institucionales o temáticos (vía verde), son las dos estrategias propuestas para su desarrollo. En el caso de los RIS (Repositorios Institucionales), son en general las universidades y los organismos de investigación los encargados de su gestión, así como de promover el depósito de la producción científica que cuente con los debidos permisos, ya sea de manera voluntaria por parte de su comunidad de investigadores, o a través de mandatos de autoarchivo.

Según datos recientes basados en la fuente BASE (Bielefeld Academic Search Engine), y publicados en el blog The Imaginary Journal of Poetic Economics, el contenido en repositorios de acceso abierto a escala global estaría superando los 100 millones de documentos (Morrison, 2016). Por otra parte, las estadísticas del directorio OpenDOAR señalan que desde octubre de 2008 a septiembre de 2016 el número de repositorios a nivel mundial se incrementó en un 198\%. Sin embargo, el desarrollo es dispar y estaría influenciado por diversos factores como la infraestructura, el idioma, la cultura, las políticas, y los mandatos (Pinfield et al., 2014).

Entre las motivaciones por las que los investigadores podrían querer difundir sus trabajos en estas plataformas (redes sociales académicas y repositorios) está el beneficio que les traería aparejada una mayor visibilidad de su producción. Algunos estudios señalan que hacer visible las publicaciones e interactuar en las redes sociales tiene efectos positivos sobre el impacto de la investigación (Bik y Goldstein, 2013; Niyazov et al., 2016). Aunque en este aspecto existen mayores discrepancias, hay trabajos que muestran que los artículos en acceso abierto autoarchivados en repositorios recibirían más citas que los que no lo están (Kullman, 2014). Es quizá por ello que estos espacios de difusión se han convertido en las plataformas "vedette" para dar visibilidad y acceso a la producción científica. Los primeros tienen como principal misión conectar investigadores y ofrecerles un espacio donde socializar los resultados de sus investigaciones, y los segundos, brindar acceso abierto a las publicaciones y garantizar su preservación a largo plazo. Podría pensarse que ambos espacios "compiten" para lograr que los investigadores depositen y difundan sus trabajos en ellos. Una reciente encuesta sobre preferencias de investigadores de la University of Rhode Island los muestra como servicios complementarios (Lovett et al., 2017).

Por otra parte, se debe considerar que los investigadores desarrollan sus actividades bajo dos principios ordenadores diferentes; uno que opera desde las instituciones académicas, y otro que lo hace desde las comunidades disciplinares. La institución comprende las instalaciones, las autoridades administrativas, y establece asimismo unas reglas del trabajo a desempeñar perteneciendo a ellas. Por su parte, los investigadores tienen además una fuerte pertenencia a su disciplina, que trasciende a la universidad y que genera un sentido de identidad entre sus miembros (Friedberg y Musselin, 1996; Clark, 1996; Fernández y Graciano, 2007). Desde esta perspectiva este trabajo parte de la hipótesis de que la difusión de la producción científica de los docentes-investigadores en los repositorios de la universidad estaría vinculada al marco institucional, en tanto que su presencia en redes sociales académicas como ResearchGate respondería al principio de pertenencia a la comunidad disciplinar.

Los indicios sobre las preferencias de los investigadores y prácticas institucionales/ disciplinares a la hora de difundir y socializar los resultados de investigación constituyen un aporte de información clave para la gestión de información de investigación. 
Tanto para las universidades como para las agencias de ciencia y tecnología, el monitoreo de las prácticas reales aporta información útil para el análisis de las políticas de mandatos, así como también para la toma de decisiones en torno a los procesos de agregación, curado y utilización de metadatos en los CRIS (Current Research Information System), los RIS (Repositorios Institucionales) y los sistemas de perfiles académicos institucionales. Asimismo, en el escenario actual que plantea nuevos desafíos en torno a la comunicación científica, contribuye a responder interrogantes en cuanto al rol que desempeñan los propios investigadores en la visibilidad y búsqueda de impacto de sus producciones.

\section{Objetivos}

Este trabajo se propone como objetivo principal realizar un aporte al conocimiento de las preferencias y prácticas que tienen los docentes-investigadores de instituciones universitarias al momento de difundir y socializar sus publicaciones en repositorios institucionales de acceso abierto y en redes sociales académicas.

Como objetivo secundario se propone establecer si existen relaciones entre la presencia, el volumen y la cobertura temporal de las producciones depositadas en estas plataformas en relación con la pertenencia institucional y áreas disciplinares, el género, el grupo etario, la formación académica y la categoría de investigador.

\section{Antecedentes}

El interés por conocer la presencia de investigadores de instituciones académicas y de investigación en redes académicas se refleja en varios estudios encontrados en la literatura reciente (Campos-Freire, Rivera Rogel y Rodríguez, 2014; Dafonte-Gómez, Míguez-González y Puentes-Rivera, 2015; González-Díaz, Iglesias-García y Codina, 2015; Campos-Freire y Rúas-Araújo, 2016; Thelwall y Kousha, 2017; Lovett et al., 2017). Una encuesta realizada a 3.500 investigadores de 95 países señala a ResearchGate como el segundo sitio web más visitado por los científicos luego de Google Académico (Van Noorden, 2014). El porcentaje de presencia de la comunidad es dispar según las instituciones y países, aunque se acerca al 50\%. ResearchGate destaca por el predominio de documentos recientes, y por su cobertura multidisciplinar, excepto en las áreas de las Humanidades y las Ciencias Sociales, donde se observa una preferencia por Academia.edu. Respecto al género, uno de los estudios realizado en una universidad noruega muestra que las mujeres tienen menor presencia en la mayoría de los servicios, y son los profesores del género masculino y de mayor edad los más representados (Mikki et al., 2015); mientras que un estudio en universidades de Portugal demuestra que la participación femenina es superior (Puentes-Rivera, Direito-Rebollal y Lago Vázquez, 2015).

En el caso de instituciones argentinas, un estudio exploratorio realizado por Boeris y Miguel (2016) sobre la presencia en redes sociales académicas de investigadores y becarios del IAR (Instituto Argentino de Radioastronomía), revela una preferencia por ResearchGate ( $48 \%$ ) respecto de Academia.edu (33\%), al tiempo que destaca una presencia relativa mayor por parte de las mujeres que de los hombres en el caso de los investigadores, y a la inversa en el caso de los becarios. Otro estudio, realizado por Enríquez et al. (2015), muestra que en la Universidad Nacional de La Plata habría una preferencia por ResearchGate sobre Academia.edu. Este último trabajo aporta datos generales extraídos de las estadísticas de la propia fuente, pero no realiza un estudio detallado de los perfiles de investigadores, como tampoco del volumen ni de la cobertura temporal de las publicaciones. En lo que respecta al desarrollo de 
repositorios de acceso abierto, en OpenDOAR, en febrero de 2017, había consignados un total de 3.320 repositorios, 2.836 (85,4\%) institucionales y $297(8,9 \%)$ disciplinares, de los cuales $354(10,7 \%)$ corresponden a América Latina, Si se comparan los valores con una medición similar realizada en el año 2012 (Molfino y González, 2012), se observa un crecimiento total para América Latina de $74,4 \%$, con algunos países que crecieron significativamente como Perú (215,4\%), Colombia (120\%), Argentina (105\%) y Chile (100\%), mientras que otros manifiestan un crecimiento, si bien menor, igualmente sustantivo como Ecuador $(52,9 \%)$ y Brasil $(48,4 \%)$. Sin embargo, el crecimiento en la cantidad de repositorios no parece vincularse directamente con la cantidad de trabajos depositados en ellos. En un estudio reciente sobre el caso español, Borrego (2016) encuentra que solo el $14 \%$ de los artículos producidos con fondos públicos de investigación se encuentran disponibles en RI. Apenas el 11\% de los artículos publicados por las universidades españolas más prestigiosas se encuentra disponible en sus propios repositorios institucionales, en tanto que más de la mitad de dichos artículos están disponibles en texto completo en ResearchGate. Datos publicados en junio de 2015 en el blog Social Media en Investigación, advierten cuanto menos comportamientos diferenciados por universidad. En la Universidad de Barcelona, el $40 \%$ de los investigadores tiene perfil en ResearchGate y el volumen de publicaciones de dicha institución en la red es 50\% mayor que la que registra el repositorio. En la Universidad Complutense de Madrid, el 70\% de la planta de docencia e investigación tiene presencia en aquella red social, con un volumen de publicaciones equivalente al de su propio repositorio. En cambio, en la Universidad Autónoma de Barcelona, con un $80 \%$ de la plantilla presente en ResearchGate, el repositorio registra un $80 \%$ más de producción de sus investigadores que en la red social.

En el caso argentino, una encuesta realizada en 2010 por el Ministerio de Ciencia, Tecnología e Innovación Productiva (Bongiovani y Nakano, 2010), mostraba una baja tasa de autoarchivo en repositorios por parte de los investigadores, siendo la tarea de depósito realizada mayormente por terceros, principalmente bibliotecarios. Sin embargo, la aprobación de la Ley 26.899 sobre los Sistemas Nacionales de Datos y Repositorios, en 2015, y su reglamentación en noviembre de 2016 (Res. 753 E/2016, Boletín Oficial, 16 de noviembre de 2016), que obliga a las instituciones a desarrollar repositorios y a los investigadores a depositar en ellos su producción, podría modificar esta situación.

\section{Materiales y métodos}

Desde una aproximación bibliométrica/cienciométrica se estudió la presencia de la comunidad académica de tres facultades de la Universidad Nacional de La Plata (UNLP), así como el volumen y la cobertura temporal de su producción científica depositada en los repositorios institucionales (RIS) de la propia universidad y en la red ResearchGate (RG). Al momento de realizar el estudio, en la UNLP existían tres RIS: SEDICI (Servicio de difusión de la producción intelectual de la UNLP), como repositorio central), Naturalis (Repositorio institucional de la Facultad de Ciencias Naturales y Museo), y Memoria Académica (Repositorio institucional de la Facultad de Humanidades y Ciencias de la Educación). La población estudiada comprende 565 docentes-investigadore ${ }^{1}$ de las facultades de Ciencias Exactas (FCE), Ciencias Naturales y Museo (FCNyM) y Humanidades y Ciencias de la Educación (FAHCE), que conforme a las estadísticas oficiales de la propia universidad son las que registran más número de docentes-investigadores, mayor cantidad de proyectos de investigación y más volumen de producción científica (http://www.unlp.edu.ar/indicadores UNLP). Para determinar la población a estudiar se establecieron los siguientes criterios: 1) ser docente de la UNLP con categoría en el programa de incentivos a la investigación, 2) tener dedicación exclusiva a la investigación, incluyendo los casos establecidos en
12. Desde el año 1993 rige en Argentina un programa de promoción de la investigación denominado Programa de Incentivos a Docentes Investigadores de Universidades Nacionales. Por tanto, desde esa fecha se tiene en cuenta la figura de docente investigador para categorizar a todos los docentes universitarios que además dirigen y/o participan de proyectos de investigación acreditados por el Ministerio de Educación de la Nación. 
el Art. 25 del Manual de Procedimientos del Programa de Incentivos a los DocentesInvestigadores de las Universidades Nacionales de Argentina, y 3) tener curriculum vitae (CV) en el sistema de Currículum Vitae Argentino (CVAR).

\section{Variables}

»Institución de pertenencia: FAHCE, FCNyM, FCE

》 Género: Masculino/Femenino

" Grupo etario: Menor de 30, 30-39, 40-49, 50-59 y 60 o más años (corresponde a las categorías definidas en el CVAR.

» Grado académico: Grado, Especialización, Maestría, Doctorado, Postdoctorado (corresponde al máximo grado de formación académica alcanzado declarado en el CVAR).

» Categoría de investigador: I, II, III, IV, V (categoría obtenida por los docentes de las Universidades Nacionales Argentinas en el Programa de Incentivos a la investigación del Ministerio de Educación de la Nación, donde la categoría V es la de ingreso y la I la más alta de la escala).

»Presencia en los RIS y en RG: SI/NO (se considera que un docente investigador tiene presencia en los repositorios institucionales de la universidad, si se localiza al menos una publicación de su autoría. En el caso de ResearchGate, si tiene perfil público y al menos una publicación de su autoría incluida en el perfil).

"Número de documentos: Número de documentos de autoría del docente investigador, encontrados en las plataformas.

» Año i: año de mayor antigüedad entre los documentos del docente investigador encontrados.

»Año f: año de mayor actualidad entre los documentos del docente investigador encontrados.

\section{Fuentes consultadas y recolección de los datos}

El listado de los docentes-investigadores que conforman la población estudiada fue provisto por las Secretarías de Investigación de las facultades. Los datos de las variables de género, grupo etario, grado académico y categoría de investigador se obtuvieron de la consulta a los curriculum vitae CVAR de los docentes-investigadores. Para la recolección de los datos de las otras variables se realizaron consultas en los RIS de la UNLP y en RG. En todos los casos las búsquedas se realizaron por el nombre y apellido del docente investigador. En el caso de la FCNyM y de la FAHCE, que tienen repositorio a nivel de la facultad, las búsquedas se realizaron tanto en el repositorio propio como en el central de la universidad (SEDICI). Para la FCE, que no tiene repositorio propio, se buscó solo en el SEDICI. La recolección de los datos se realizó durante el primer semestre de 2016.

\section{Análisis de los datos}

Se estudió la presencia de la comunidad académica en los RIS y en RG desde la perspectiva de la institución de pertenencia, género, grupo etario, grado académico y categoría de investigador. La presencia se determinó a partir del número y porcentaje de docentes-investigadores registrados en cada plataforma, y el volumen de producción a partir del recuento de sus publicaciones depositadas en ellas. A partir de este último dato, se calcularon la media de la cantidad de documentos por docente investigador y la desviación típica, así como la cobertura temporal a partir de la diferencia entre el año $f$ y el año i de las publicaciones registradas por docente investigador. Además, se analizó la estructura de las relaciones entre las variables antes mencionadas, a partir de un Análisis de Correspondencias Múltiples (ACM), técnica estadística multivariada que permite reducir la dimensionalidad de los datos 
de variables categóricas, y realizar una interpretación de las tendencias predominantes en relación al fenómeno estudiado. Cabe aclarar que para este último análisis quedaron excluidas las variables de género y cobertura temporal por el bajo poder de discriminación observada.

\section{Resultados}

\section{Caracterización de la población}

De la totalidad de docentes-investigadores (565), el 45\% (256) pertenecen a la Facultad de Ciencias Exactas (FCE), el 35\% (197) a la Facultad de Ciencias Naturales y Museo (FCNyM) y el $20 \%$ a la Facultad de Humanidades y Ciencias de la Educación (FAHCE). En los tres casos el porcentaje de mujeres supera al de hombres, con mayor diferencia en la FCNyM que en las otras dos Facultades. Con relación a la edad, el mayor porcentaje de la población se concentra en los grupos de 40-49 y 50-59 años, edades típicas de docentes-investigadores que tienen ya una cierta trayectoria académica y poseen un mayor volumen de producción científica. Esto se condice con el grado académico alcanzado, ya que la mayoría poseen grados académicos de doctorado y postdoctorado. En lo que respecta a las categorías de investigador, el mayor porcentaje de los docentes-investigadores tiene la categoría III, seguida de la II para la FAHCE, y de las IV para las otras dos Facultades. En los tres casos es menor el porcentaje de docentes con las categorías extremas (I y V). (Tabla 1 en Anexo). Se debe agregar además, que según el área de conocimiento que seleccionaron los propios docentesinvestigadores en sus CVs, como el área disciplinar de su actividad académica, el 62\% de los pertenecientes a la FAHCE se incluye en Humanidades y el $36 \%$ en Ciencias Sociales. En las otras dos Facultades el mayor porcentaje de investigadores indicó como área principal de actividad a las Ciencias Naturales y Exactas $(82 \%$ en FCNyM y $77 \%$ en la FCE). Por lo que el estudio representa en gran medida estas grandes áreas disciplinares.

\section{Presencia de los docentes-investigadores en los RIS y en RG}

En las tres Facultades hay una más alta presencia de docentes-investigadores en los RIS que en la red social ResearchGate. En cuanto a ResearchGate son notables las diferencias entre las Facultades: en la FCE y en FCNyM más del 60\% de los investigadores tiene un perfil público en esta red social, en tanto que en la FAHCE apenas alcanza el 16\% (Tabla 2 en Anexo). Esto es coincidente con hallazgos de estudios previos que muestran menor presencia de disciplinas socio-humanísticas en esta red social respecto de otras disciplinas como las de exactas y naturales (Campos-Freire, Rivera Rogel y Rodríguez, 2014; Campos-Freire y Rúas-Araújo, 2016; Thelwall y Kousha, 2017).

Cuando se compara el volumen de la producción depositada, las dos Facultades con RIS propio tienen un promedio de documentos por docente investigador más elevado que en el repositorio central. En el caso de la FCE el valor de esta variable en el RIS central es notablemente bajo. Por otro lado, destaca el hecho de que en las tres Facultades el número medio de documentos por docente investigador es mayor en la red social que en los RIS, por lo que sería posible pensar que el tipo de servicio que ofrecen las plataformas, así como los niveles de granularidad, podrían influir al momento de la elección sobre dónde depositar.

En términos de la cobertura temporal de los documentos se observa una mayor amplitud en RG para el caso de la FCE, cuyos valores medios de primer y último año de los documentos son 1996 y 2015, respectivamente; mientras que su cobertura en el repositorio central de la universidad abarca un período más acotado, con una 
amplitud de 5 años (2004-2009). La FCNyM presenta una mayor cobertura en años en el repositorio propio (1998-2014) y luego en la red social (1999-2015), y notablemente menor en el repositorio central (1999-2009). En ambos casos, el repositorio central registra una menor actualidad de los documentos. La FAHCE, en cambio, presenta una cobertura similar en los repositorios (2002-2014 en Memoria Académica y 2001-2013 en SEDICI), y más acotado en la red social (2005-2012).Debe tenerse en cuenta, que aunque la edad como la trayectoria del docente investigador puedan tener influencia sobre esta variable, lo que resalta son las diferencias entre plataformas para la misma población.

Hay una mayor presencia de mujeres en todas las plataformas, excepto en el caso de la FAHCE en ResearchGate (Tabla 3 en Anexo). Esto coincide con algunos estudios previos (Puentes-Rivera, Direito-Rebollal y Lago Vázquez, 2015; Dafonte-Gómez, Míguez-González y Puentes-Rivera, 2015; Boeris y Miguel, 2016), y se debe además a una mayor representación del género femenino en la población estudiada. Sin embargo, el promedio de documentos por docente investigador es superior para los hombres que para las mujeres, exceptuando el caso de la FAHCE en el RIS propio. Un factor a considerar es que, aunque existen diferencias entre disciplinas, hay estudios que muestran que los hombres alcanzan índices de productividad de las publicaciones más elevados que las mujeres (Abramo et al., 2009; Torres-Salinas, Muñoz-Muñoz y Jiménez-Contreras, 2011; Kretschmer et al., 2012; Miguel et al., 2013), y esto podría explicar este resultado. Otro dato que destaca es que tanto hombres como mujeres registran una media de documentos más elevada en el repositorio propio de la facultad que en el central de la universidad, y no se observan diferencias por género en relación con la cobertura temporal de los documentos, que sigue la tendencia general.

Los docentes-investigadores de los grupos de 40-49 y 50-59 son los que tienen mayor presencia tanto en los RIS como en RG (Tablas 4a-c en Anexo). Esto coincide con las características de la población estudiada, que se concentra mayormente en estos grupos. Sin embargo, cuando se analiza el número medio de documentos por docenteinvestigador, tanto en los repositorios como en la red social es posible apreciar que este valor aumenta con la edad, siendo los del grupo de 60 o más los que tienen en promedio más documentos difundidos en estas plataformas; y ese valor va decreciendo a medida que disminuye la edad. Una excepción se da en el caso de RG en la FAHCE, pero se debe a un solo caso en el grupo etario 30-39 con una elevada cantidad de documentos.

En cuanto a la cobertura temporal de la producción, se evidencia que a medida que aumenta la edad de la población se difunden publicaciones más antiguas (año i). Por otra parte, cuando se analiza la actualidad (año f), es en la red social donde se registran los documentos más recientes. Una excepción se da para la FAHCE, pero dada su escasa presencia en esta red no es posible realizar interpretaciones generales en este aspecto.

En las distintas plataformas estudiadas y en las tres Facultades hay una mayor presencia de docentes-investigadores con doctorados y postdoctorados, observando este fenómeno también para la variable volumen de documentos (Tablas 5a-c en Anexo). Esto coincide con resultados de otros estudios donde los investigadores de mayor jerarquía académica y mayor edad son los que tienen una mayor representación en las redes académicas (Mikki et al., 2015). Cabe aclarar una diferencia observada en el caso de la FAHCE en RG, pero nuevamente aquí la escasa presencia de sus investigadores en RG dificulta la identificación de patrones y tendencias del comportamiento de difusión y socialización de la producción de esta comunidad académica en la red social. 


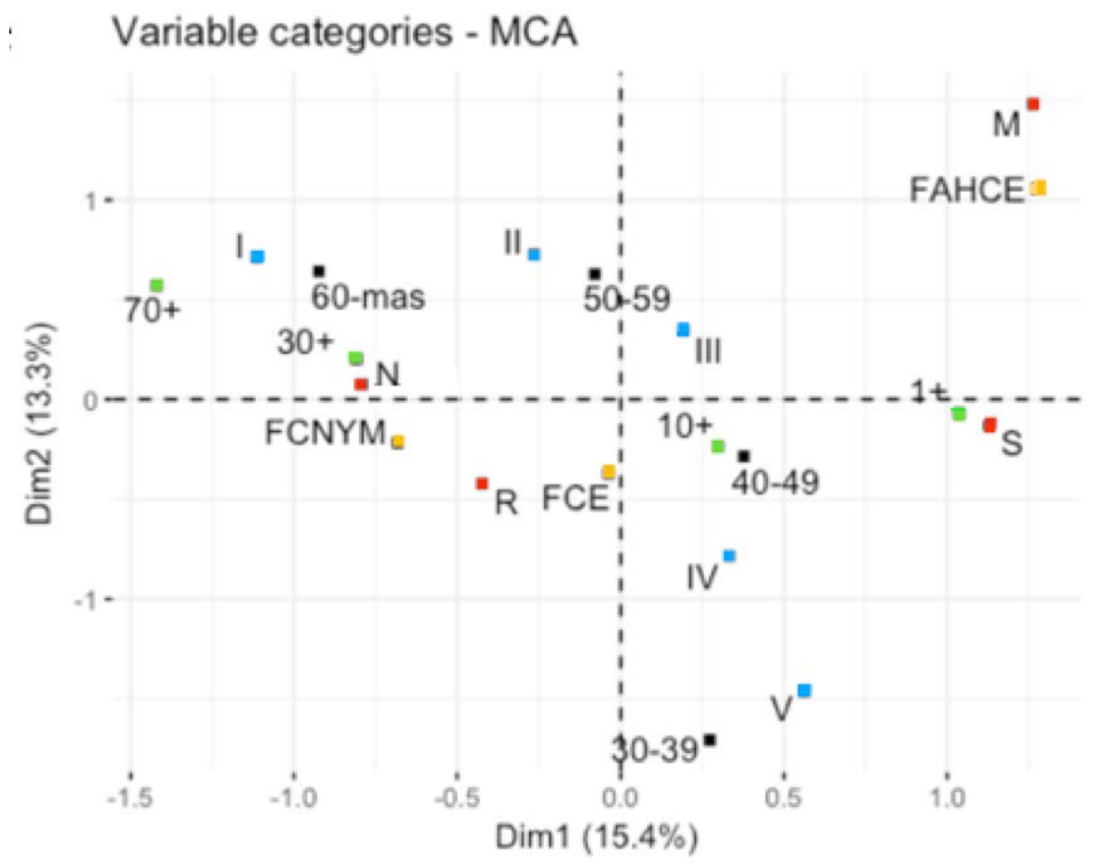

amarillo: facultades

negro: grupo etario.

rojo: repositorios

ACM: Análisis de correspondencias múltiples.

verde: producción

Para las restantes referencias, véase el texto

celeste: categorías en el programa de incentivos;

(alternativa: véase Materiales y métodos)

Figura 1. Estructura de relaciones entre variables (ACM)

Considerando la categoría de investigador en el programa de incentivos, en todos los casos se mantiene una relación proporcional entre la distribución de la población por categorías y la presencia de los docentes-investigadores en las distintas plataformas. Así, la mayor presencia corresponde a los que tienen categoría III, seguido de la IV en las FCNyM y FCE, y categoría II en el caso de la FAHCE (Tablas 6a-c en Anexo). Sin embargo, cuando se analiza la media de documentos por docente- investigador, este valor sigue una distribución de orden decreciente por categoría, siendo mayor para la categoría I y menor para la V. Esto se da en las tres Facultades, de manera más pronunciada en las que tienen RIS propio, respecto del repositorio central. Se observa el mismo patrón en ResearchGate para la FCNyM y FCE. También se da para estas dos facultades que la media de edad de los documentos incluidos en las plataformas aumenta a medida que se incrementa la categoría del docente investigador. Es decir, los docentes-investigadores con categoría I tienen documentos más antiguos tanto en los RIS como en RG, y ese valor medio desciende a medida que decrece la categoría. Por otro lado, los documentos más recientes son de docentes-investigadores de la FCNyM y la FCE, independientemente de la categoría.

Como complemento del análisis univariado, la aplicación de la técnica de análisis multivariado de ACM arroja resultados sobre la estructura de relaciones que resulta de considerar de manera conjunta la institución de pertenencia de los docentesinvestigadores (amarillo), el grupo etario (negro), la categoría de investigador (celeste), la cantidad de documentos depositados (verde), y la presencia en los RIS y en RG (rojo) (Figura 1) 
La dimensión 1 (eje horizontal) discrimina principalmente a los docentes-investigadores por la institución de pertenencia y por su mayor o menor presencia en las plataformas. Así, la FAHCE se asocia principalmente a su repositorio Memoria Académica (M) como también al repositorio central SEDICI (S), ubicándose ambos en el extremo derecho del eje horizontal. Por otro lado, la FCNyM se ubica a la mitad izquierda de dicho eje, asociada a su repositorio Naturalis (N) y a RG (R). La FCE aparece también en este sector, aunque más hacia el centro del eje y más asociada a RG (R). La dimensión 2 (eje vertical), discrimina por grupo etario, categoría de investigador y cantidad de documentos. Aquí puede verse que los más jóvenes, con categorías inferiores y con menos cantidad de documentos se ubican en la parte inferior del eje, mientras que los docentes-investigadores de mayor edad, categorías más altas y mayor cantidad de trabajos depositados se posicionan en la parte superior del mismo.

En la Figura 2 se puede apreciar con más detalle el caso particular de la variable presencia en RIS y RG. La elipse que encierra a los puntos verdes ubicada en el extremo superior derecho de la figura corresponde a los documentos depositados en Memoria Académica (M) por los investigadores de la FAHCE. Las elipses que encierran a los puntos naranjas (Naturalis) y violetas (RG) están alejadas de la anterior y muestran un marcado grado de solapamiento. Representan a los investigadores de la FCNYM, que tienen presencia tanto en su propio repositorio (Naturalis) como en RG, y a los docentes-investigadores de FCE, que depositan principalmente sus artículos en RG. SEDICI se ubica entre los dos grupos de elipses (verde y naranja + violeta), solapándose algunos de sus documentos con los de aquellas. Esto obedece fundamentalmente a que en dicho repositorio se incluyen publicaciones de docentes-investigadores de las tres facultades analizadas (Tabla 2). Así, en relación con las plataformas estudiadas, los investigadores de las facultades de ciencias exactas y de ciencias naturales se asemejan más en sus prácticas de difusión y socialización, y se diferencian de los de ciencias sociales y humanidades.

\section{Conclusiones y discusión}

Los resultados obtenidos en este trabajo permiten visualizar nuevas dimensiones para el análisis de las preferencias y prácticas de difusión y socialización de la producción científica en repositorios institucionales y en las redes sociales académicas.

Por un lado, se observa que el nivel de presencia en estas plataformas de los docentes-investigadores con dedicación exclusiva es alto, superando el $80 \%$, y que existe una mayor presencia en los repositorios institucionales (RIS) que en la red social académica ResearchGate (RG). Sin embargo, la media de documentos por docente investigador es mayor en RG que en los RIS, incluso en el caso de la facultad de Humanidades, cuya comunidad tiene muy baja presencia en la red social. Esto podría deberse a que en los repositorios no necesariamente la producción es depositada por los investigadores, que en algunos casos ignoran su existencia, pudiendo ser registrada por terceros, o provenir de la cosecha de datos de otros servicios. Por otro lado, el hecho de que cada uno individualmente deposite más en RG, lo cual es claramente una práctica individual, hace pensar que esta red social académica les resulta a los docentes-investigadores más atractiva que los RIS, quizá por presentar procedimientos de depósito más ágiles, o por sus funcionalidades comunicativas y/o provisión de métricas de impacto de la producción.

Por otro lado, la mayor presencia en el RIS de la Facultad a la que pertenece el docente investigador, frente al repositorio central de la universidad, podría deberse a que a la hora de elegir un repositorio donde autoarchivar la producción, los docentesinvestigadores lo hacen en aquel más afín a su comunidad (si se considera que el 


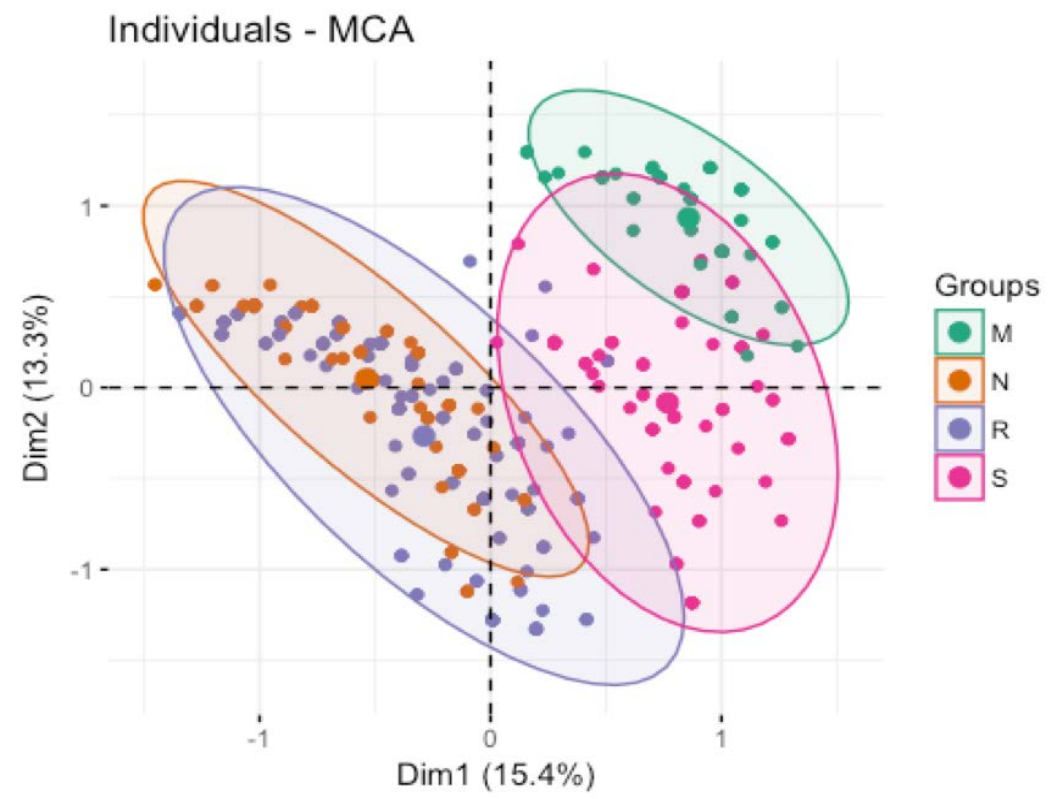

verde (M): Memoria Académica (FAHCE) naranja (N): Naturalis (FCNYM)

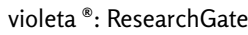
fucsia (S): SEDICl

Figura 2. Plano factorial de la variable Presencia en RIs y RG

depósito es realizado por ellos mediante el proceso de autoarchivo); caso contrario, si el depósito es realizado por terceros (bibliotecarios u otro personal a cargo del repositorio) podría indicar una mayor capacidad de las Facultades para obtener los documentos y registrar la producción de su plantel docente investigador. Es importante mencionar que los repositorios de la UNLP solo tienen mandatos de autoarchivo para los trabajos de fin de carrera (tesis de grado o de posgrado). Aunque hay estudios que revelan que los mandatos no garantizan cambios en el comportamiento de dicha práctica por parte de los investigadores (Xia et al., 2012), la reciente reglamentación de la Ley 26.899 de Creación de Repositorios Digitales Institucionales de Acceso Abierto, sancionada en 2013 por el Senado de la Nación Argentina (BO, 16 de noviembre de 2016), que establece la obligatoriedad del depósito de la producción científico-técnica en repositorios institucionales, hace pensar que en un futuro no muy lejano podría cambiar la visibilidad de la producción en los repositorios locales.

Respecto a la actualidad de los documentos depositados, son más actuales los de RG que los de los RIS. Si se tiene en cuenta que en RG las publicaciones más recientes reciben más visitas que las más antiguas (Thelwall y Kousha, 2017), esto podría ser una motivación para que los docentes-investigadores suban sus últimas publicaciones a la red social con mayor prontitud que al repositorio institucional.

El hecho de que a mayor edad y categoría de los docentes-investigadores, mayor es el número medio de documentos visibles en los RIS y en RG, a la vez que es más amplia la cobertura temporal de los documentos respecto de la producción de los grupos más jóvenes y con categorías inferiores, no parece más que obedecer a la lógica de a mayor edad/mayor vida académica.

En cuanto al género, deberían realizarse estudios más exhaustivos para confirmar esta tendencia en la producción de los docentes-investigadores de la UNLP, haciendo extensivo el estudio a más Facultades y abarcando otras disciplinas. 
Por último agregar, que en las prácticas de difusión y socialización de la producción científica en repositorios institucionales y redes sociales académicas hay que considerar una diversidad de aspectos que se encuentran atravesados por acciones individuales (caso RG) o por acciones individuales/institucionales (caso autoarchivo y promoción en RIS); y que dichas acciones, además, no escapan a las trayectorias académicas y de investigación que se alcanzan en el marco de las instituciones, lo cual se conjuga con las tradiciones disciplinares de las comunidades de cada Facultad estudiada. De modo que, es posible reafirmar la idea del doble principio ordenador que atraviesa a las instituciones académicas, el cual se refleja también en las prácticas de comunicación científica de sus comunidades. En este sentido, y siguiendo la hipótesis planteada en este estudio los resultados permitirán afirmar que la red social como espacio de interacción y socialización responde mejor a los intereses de pertenencia a una comunidad disciplinar que los RIS, que siguen la lógica diferente basada en la pertenencia institucional. De igual modo, las diferencias encontradas en la presencia en RG entre las Humanidades y Ciencias sociales y las Ciencias Exactas y Naturales ponen de relieve una vez más que el uso de estas plataformas como espacios de difusión de la producción difiere según el campo disciplinar.

\section{Agradecimiento}

Los autores agradecen a la Bib. Doc. Lorena Caprile por su colaboración en la etapa de revisión de antecedentes y en la recolección de datos. 


\section{Q Referencias Bibliográficas}

》 Abramo, G.; C.A. D’Angelo y A. Caprasecca. 2009. Gender differences in research productivity: a bibliometric analysis of the Italian academic system. En Scientometrics. Vol.79, no. 3, 517-539.

"Alba-Ruiz, Rubén; Trinidad Bullejos de la Higuera; Clara Bermudez-Tamayo; Manuel Colmenero-Ruiz; Carmen Martínez Valero; Manuela Expósito Ruiz y M. M. Rodríguez del Aguila. 2011. Estrategias de difusión de la actividad investigadora en un centro hospitalario. Trabajo presentado a las XIV Jornadas Nacionales de Información y Documentación en Ciencias de la Salud, realizado en Cádiz del 13 al 15 de abril de 2011. <http://eprints.rclis.org/15935/> [Consulta: 19 agosto 2017].

» Bik, H. M. y M. C. Goldstein. 2013. An introduction to social media for scientists. En PlosBiology. Vol. 1, no. 4. <e1001535. doi.org/10.1371/journal.pbio.1001535> [Consulta: 15 septiembre 2017].

"Boeris, Claudia y Sandra Miguel. 2016. Perspectivas de la aplicación de altmetrías en el análisis de los perfiles de investigadores del IAR. Trabajo presentado al Workshop Científico \& Tecnológico Radioastronomía en la Argentina. 50 años del $I A R$, realizado en La Plata del 28 al 29 de marzo de 2016.

» Borgman, Christine. 1989. Bibliometrics and scholarly communication: editor`s introduction. En Communication Research. Vol. 16, no. 4, 583-599.

»Björk, Bo-Christer. 2007. A model of scientific communication as a global distributed information system. En Información Research. Vol. 12, no. 2. <http:// www.informationr.net/ir/12-2/paper307.html> [Consulta: 20 noviembre 2016].

"Bongiovani, Paola y Silvia Nakano. 2010. El Sistema Nacional de Repositorios Digitales (SNRD): la experiencia de articulación y coordinación institucional de los repositorios digitales en ciencia y tecnología. Trabajo presentado a la Jornada Virtual de Acceso Abierto Argentina, realizada en Buenos Aires el 21 de octubre de 2010. <http://www.caicyt-conicet.gov.ar/micrositios/accesoabierto/ wp-content/uploads/2016/o6/Ponencia-Bongiovanni-Nakano.pdf> [Consulta: 15 de agosto de 2017].

"Borrego, Ángel. 2016. Measuring compliance with a spanish government open access mandate. En Journal of the Association for Information Science and Technology. Vol. 67, no. 4, 757-764. Doi: <10.1002/asi.23422>.

»BOAl. Budapest Open Access Initiative. 2002. Budapest: Open Society Institute. $<$ http://www.budapestopenaccessinitiative.org/read> [Consulta: 20 noviembre 2017].

» BOAI. Budapest Open Access Initiative 2015. Budapest: Open Society Institute. <http://www.budapestopenaccessinitiative.org/boai15-1> [Consulta: 20 noviembre 2017].

»Campos-Freire, F. y J. Rúas-Araújo. 2016. Uso de las redes sociales digitales profesionales y científicas: el caso de las tres universidades gallegas. En El Profesional de la Información. Vol. 25, no. 3, 431-440. <http://dx.doi.org/10.3145/ epi.2016.may.13> [Consulta: 7 abril 2017].

" Campos-Freire, F.; D. Rivera Rogel y C. Rodríguez. 2014. La presencia e impacto de las universidades de los países andinos en las redes sociales digi- 
tales. En Revista Latina de Comunicación Social, Vol. 69, 571-592. Doi: <10.4185/ RLCS-2014-1025>.

»Clark, B. 1996. El sistema de educación superior. Una visión comparativa de la organización académica. México: Nueva Imagen.

»Dafonte-Gómez, Alberto; María Isabel Míguez-González e Iván Puentes-Rivera. 2015. Redes sociales académicas: presencia y actividad en Academia.edu y ResearchGate de los investigadores en comunicación de las universidades gallegas. Trabajo presentado a 1oth Iberian Conferenceon Information Systems and Technologies (CISTI) realizado en Aveiro del 17 al 20 de Junio de 2015.

"Echavarría Ramírez, Andrés Felipe. 2010. Redes sociales académicas... el boom de la Web 2.0 académica. En Blog de la Universidad ICESI. <http://www.icesi. edu.co/blogs/egatic/tag/redes-sociales-academicas/> [Consulta: 15 marzo 2017].

»Enríquez, Silvia C.; Sandra Gargiulo; Enrique Verdecia Carballo y Norberto Wenk. 2015. Circulación de textos científicos en sitios web académicos no institucionales. Trabajo presentado a las III Jornadas de TIC e Innovación en el Aula, realizado en La Plata el 7 de septiembre de 2015. <http://hdl.handle. net/10915/48652> [Consulta: 20 agosto 2017].

»Fernández, Laura y Corina Graciano. 2007. Producción y difusión del conocimiento en la Facultad de Ciencias Naturales de la Universidad Nacional de La Plata - Estrategias de posicionamiento de los grupos. Trabajo presentado al $\mathrm{V}$ Encuentro Nacional y II Latinoamericano: I Universidad como objeto de investigación, realizado en Tandil del 30 de agosto al 1 de septiembre de 2007. Universidad Nacional del Centro de la Provincia de Buenos Aires.

» Friedberg, Erhard y Christine Musselin. 1996. La noción del sistema universitario y sus implicancias para el estudio de las universidades. México: Universidad Futura.

" Garvey, William D. y Belver C. Griffith. 1972. Communication and information processing within scientific disciplines: Empirical findings for Psychology. En Information Storage and Retrieval. Vol. 8, no. 3, 123-136.

" González Díaz, Cristina; Mar Iglesias-García y Luis Codina. 2015. Presencia de las universidades españolas en las redes sociales digitales científicas: caso de los estudios de comunicación. En El Profesional de la Información. Vol. 24, no. 5, 640-647.

»Hurd, Julie M. 2000. The transformation of scientific communication: a model for 2020. En Journal of the Association for Information Science and Technology. Vol. 51, no. 14, 1279-1283.

»Kretschmer, Hildrun; Alexander Pudovkin y Johannes Stegmann. 2012. Research evaluation. Part II: gender effects of evaluation: are men more productive and more cited than women? En Scientometrics. Vol. 93, no. 1, 17-30.

» Kullman, L. 2014. The effect of Open Access on citation rates of self-archived Articles at Chalmers. En IATUL 2014 - Annual Conference ( $35^{\circ}: 2$ al 5 de junio de 2014: Espoo). Trabajos presentados. Espoo, Finland: Aalto University. <http:// publications.lib.chalmers.se/records/fulltext/198512/local_198512.pdf> [Consulta: 15 septiembre 2017].

» Liu, Ziming. 2003. Trends in transforming scholarly communication and their implications. En Information Proccessing and Management. Vol. 39, no. 6, 889-898.

»Lovett, Julia A.; Andrée J. Rathemacher; Divana Boukari y Corey Lang. 2017. Institutional Repositories and Academic Social Networks: Competition or Com- 
plement? A study of Open Access policy compliance vs. ResearchGate participation. En Journal of Librarianship and Scholarly Communication. No. 5 (General Issue). eP2183 <https//doi.org/10.7710/2162-3309.2183> [Consulta: 20 octubre 2017].

» Miguel, Sandra; Mónica Hidalgo; Edgardo Stubbs; Paula Posadas y Edgardo Ortiz Jauréguizar. 2013. Estudio bibliométrico de género en la paleontología de vertebrados. El caso de la revista argentina Ameghiniana (1957-2011). En Investigación Bibliotecológica. Vol. 27, no. 61, 133-155.

» Mikki, S.; M. Zygmuntowska; Ø. L. Gjesdal y H. A. Al Ruwehy. 2015. Digital presence of norwegian scholars on academic network sites-Where and Who Are They? En PLoSONE. Vol. 10, no. 11, e0142709. Doi: <10.1371/journal.pone.0142709> [Consulta: 20 octubre 2017].

"Molfino, María del Rosario y Claudia González. 2012. Acceso Abierto a la literatura científica y a los datos de investigación: escenario de oportunidad para Latinoamérica. Trabajo presentado en II ETHICOMP Latinoamérica, realizado en Bahía Blanca del 8 al 12 de octubre de 2012. <http://www.memoria.fahce.unlp. edu.ar/trab_eventos/ev.2971/ev.2971.pdf> [Consulta: 20 octubre 2017].

" Morrison, Heather. 2016. Dramatic growth of Open Access. En The Imaginary Journal of Poetic Economics. <http://poeticeconomics.blogspot.com.ar/2016/12/ dramatic-growth-of-open-access-december.html> [Consulta: 20 octubre 2017]

» Niyazov, Y.; C. Vogel; R. Price; B. Lund; D. Judd; A. Akil; M. Mortonson; J. Schwartzman y M. Shron. 2016. Open Access meets discoverability: citation to articles posted to Academia.edu. En Plos ONE. Vol. 11, no. 2, e0148257 Doi: $<10.1371$ /journal.pome.0148257> [Consulta: 15 septiembre 2017].

»Ortega, José Luis. 2015. Relationship between altmetric and bibliometric indicators across academic social sites: The case of CSIC's members. En Journal of Informetrics, Vol. 9, 39-49.

"Pinfield, S.; J. Salter; P. A. Bath; B. Hubbard; P. Millington; J. H. S. Anders y A. Hussain. 2014. Open-access repositories worldwide, 2005-2012: Past growth, current characteristics and future possibilities. En Journal of the American Society for Information Science and Technology. Vol. 65, no. 12, 2404-2421.

»Puentes-Rivera, Iván; Sabela Direito-Rebollal y Diana Lago Vázquez. 2015. Las redes sociales científicas: presencia y actividad de los investigadores de comunicación de las universidades de Portugal en Academia.edu y ResearchGate.net. En La pantalla Insomne. Universidad de La Laguna. <http:// www.revistalatinacs.org/15SLCS/2015_libro/137_Puentes.pdf> [Consulta: 15 octubre 2017].

»Russell Barnard, Jane. 2007. La comunicación, publicación y validación de la ciencia: Nuevos enfoques y retos. En: Tópicos de investigación en Bibliotecología y sobre la Información. Edición conmemorativa de los XXV años del Centro Universitario de Investigaciones Bibliotecológicas. Vol. I. México: Nueva Visión.

"Santana Arroyo, Sonia. 2010. Redes de intercambio de información científica y académica entre los profesionales, en el contexto de la Web 2.o. En Revista Cubana de Información en Ciencias de la Salud. Vol. 21, no. 3 <http://www.acimed. sld.cu/index.php/acimed/article/view/98> [Consulta: 12 diciembre 2017].

»Thelwall, Mike y Kayvan Kousha. 2017. ResearchGate articles: age, discipline, audience size and impact. En Journal of the Association for Information Science and Technology. Vol. 68, no. 2, 468-479. Doi: <10.1002/asi.23675>

» Torres-Salinas, Daniel; Ana Muñoz-Muñoz y Evaristo Jiménez-Contreras. 2011. 
Análisis bibliométrico de la situación de las mujeres investigadoras de Ciencias Sociales y Jurídicas en España. En Revista Española de Documentación Científica. Vol. 34, no. 1, 11-28. <http://redc.revistas.csic.es/index.php/redc/article/ view/680/754> [Consulta: 3 noviembre 2017].

"Van Noorden, R. 2014. Online collaboration: Scientists and the social network. En Nature. Vol. 512, no. 7513 <http://www.nature.com/news/online-collaboration-scientists-and-the-social-network-1.15711> [Consulta: 4 agosto 2016].

» Xia, J.; S. B. Gilchrist; N. X. P. Smith; J. A. Kingery; J. R. Radecki; M. L. Wilhelm; K. C. Harrison; M. L. Ashby y J. Alyson. 2012. A Review of Open Access selfarchiving mandate policies. En Libraries and the Academy. Vol. 12, no. 1, 85-102. 


\section{Anexo}

\begin{tabular}{|c|c|c|c|c|c|c|}
\hline & \multicolumn{6}{|c|}{ Institución } \\
\hline & \multicolumn{2}{|c|}{ FAHCE } & \multicolumn{2}{|c|}{ FCE } & \multicolumn{2}{|c|}{ FCNyM } \\
\hline Población & \multicolumn{2}{|c|}{ Nro } & \multicolumn{2}{|c|}{ Nro } & \multicolumn{2}{|c|}{ Nro } \\
\hline & \multicolumn{2}{|c|}{112} & \multicolumn{2}{|c|}{256} & \multicolumn{2}{|c|}{197} \\
\hline Género & $\#$ & $\%$ & $\#$ & $\%$ & $\#$ & $\%$ \\
\hline Femenino & 58 & 51,8 & 135 & 52,7 & 122 & 61,9 \\
\hline Masculino & 54 & 48,2 & 121 & 47,27 & 75 & 38,07 \\
\hline Grupo etario & Nro & $\%$ & Nro & $\%$ & Nro & $\%$ \\
\hline Menor de 30 & $\mathrm{o}$ & 0,0 & 0 & $\mathrm{o}$ & 1 & 0,508 \\
\hline $30-39$ & 5 & 4,5 & 42 & 16,41 & 31 & 15,74 \\
\hline $40-49$ & 44 & 39,3 & 104 & 40,6 & 66 & 33,5 \\
\hline $50-59$ & 51 & 45,5 & 84 & 32,8 & 60 & 30,5 \\
\hline 60 y más & 12 & 10,7 & 26 & 10,16 & 39 & 19,8 \\
\hline Formación académica & Nro & $\%$ & Nro & $\%$ & Nro & $\%$ \\
\hline Postdoctorado & 19 & 17,0 & 140 & 54,7 & 44 & 22,3 \\
\hline Doctorado & 60 & 53,6 & 102 & 39,8 & 126 & 64,0 \\
\hline Maestría & 16 & 14,3 & $\mathrm{o}$ & 0,0 & $\mathrm{o}$ & 0,0 \\
\hline Especialización & 2 & 1,8 & 2 & 0,8 & 3 & 1,5 \\
\hline Grado & 12 & 10,7 & 8 & 3,1 & 18 & 9,1 \\
\hline Sin datos & 3 & 2,7 & 4 & 1,6 & 6 & 3,0 \\
\hline Categoría inv & Nro & $\%$ & Nro & $\%$ & Nro & $\%$ \\
\hline I & 9 & 8,0 & 35 & 13,7 & 29 & 14,72 \\
\hline II & 26 & 23,2 & 45 & 17,6 & 31 & 15,74 \\
\hline III & 50 & 44,6 & 78 & 30,5 & 65 & 33,0 \\
\hline IV & 18 & 16,1 & 63 & 24,6 & 44 & 22,3 \\
\hline $\mathrm{V}$ & 9 & 8,0 & 35 & 13,7 & 28 & 14,21 \\
\hline
\end{tabular}

Tabla 1. Distribución de docentes-investigadores con dedicación exclusiva por institución de pertenencia, género, grupo etario, formación académica y categoría de investigador. 


\begin{tabular}{|c|c|c|c|c|c|c|c|c|c|c|}
\hline \multirow[b]{2}{*}{ Institución } & \multirow[b]{2}{*}{ Plataforma } & \multicolumn{2}{|c|}{ Docinv } & \multicolumn{2}{|c|}{ Documentos } & \multicolumn{2}{|c|}{ Año i } & \multicolumn{2}{|c|}{ Año $f$} & \multirow{2}{*}{$\begin{array}{c}\text { Cobertura } \\
\text { temporal } \\
\text { (años) }\end{array}$} \\
\hline & & Nro & $\%$ & Media & $\begin{array}{c}\text { Desv. } \\
\text { Tip. }\end{array}$ & Media & $\begin{array}{c}\text { Desv. } \\
\text { Tip. }\end{array}$ & Media & $\begin{array}{c}\text { Desv. } \\
\text { Tip. }\end{array}$ & \\
\hline \multirow{3}{*}{ FAHCE } & $\begin{array}{c}\text { Memoria } \\
\text { Académica }\end{array}$ & 107 & 95,54 & 12,91 & 10,12 & 2002 & 5,41 & 2014 & 1,86 & 12 \\
\hline & SEDICI & 111 & 99,11 & 9,50 & 6,36 & 2001 & 6,04 & 2013 & 2,64 & 12 \\
\hline & $\mathrm{RG}$ & 18 & 16,07 & 17,56 & 18,45 & 2005 & 4,55 & 2012 & 3,90 & 8 \\
\hline \multirow{3}{*}{ FCNyM } & Naturalis & 192 & 97,5 & 33,57 & 25,78 & 1998 & 7,43 & 2014 & 1,52 & 15 \\
\hline & SEDICl & 175 & 88,9 & 4,54 & 6,31 & 1999 & 10,76 & 2009 & 6,58 & 10 \\
\hline & $\mathrm{RG}$ & 125 & 63,4 & 37,79 & 31,05 & 2001 & 7,08 & 2015 & 1,02 & 14 \\
\hline \multirow{2}{*}{ FCE } & SEDICl & 207 & 80,9 & 2,17 & 1,93 & 2004 & 7,59 & 2009 & 7,09 & 4 \\
\hline & $\mathrm{RG}$ & 168 & 65,6 & 43,06 & 48,97 & 1996 & 9,05 & 2015 & 1,17 & 19 \\
\hline
\end{tabular}

Tabla 2. Presencia y producción científica de docentes-investigadores en repositorios y en ResearchGate

\begin{tabular}{|c|c|c|c|c|c|c|c|c|c|}
\hline & \multirow[b]{2}{*}{ Género } & \multicolumn{2}{|c|}{ Docinv } & \multicolumn{2}{|c|}{ Documentos } & \multirow{2}{*}{$\begin{array}{l}\text { Año i } \\
\text { Media } \\
\end{array}$} & \multirow{2}{*}{$\begin{array}{l}\text { Año f } \\
\text { Media } \\
\end{array}$} & \multirow{2}{*}{$\begin{array}{c}\text { Cobertura } \\
\text { temporal } \\
\text { (Años) }\end{array}$} \\
\hline & & & Nro & $\%$ & Media & $\begin{array}{c}\text { Desv. } \\
\text { Tip. }\end{array}$ & & & \\
\hline \multirow{6}{*}{ FAHCE } & $\begin{array}{c}\text { Memoria } \\
\text { Académica }\end{array}$ & $\mathrm{F}$ & 56 & 52,34 & 13,82 & 11,90 & 2002 & 2013 & 12 \\
\hline & & M & 51 & 47,66 & 11,90 & 7,70 & 2002 & 2014 & 12 \\
\hline & SEDICl & $\mathrm{F}$ & 58 & 52,25 & 9,47 & 7,09 & 2001 & 2013 & 12 \\
\hline & & M & 53 & 47,75 & 9,53 & 5,51 & 2001 & 2013 & 12 \\
\hline & RG & $\mathrm{F}$ & 8 & 44,44 & 14,13 & 18,04 & 2005 & 2012 & 7 \\
\hline & & $M$ & 10 & 55,56 & 20,30 & 19,27 & 2005 & 2013 & 8 \\
\hline \multirow{6}{*}{ FCNyM } & Naturalis & $\mathrm{F}$ & 118 & 62,11 & 31,77 & 23,95 & 1999 & 2014 & 13 \\
\hline & & M & 72 & 37,89 & 36,53 & 28,47 & 1998 & 2014 & 15 \\
\hline & SEDICI & $\mathrm{F}$ & 107 & 61,14 & 4,44 & 6,50 & 1999 & 2009 & 10 \\
\hline & & $\mathrm{M}$ & 68 & 38,86 & 4,71 & 6,04 & 1999 & 2009 & 10 \\
\hline & $\mathrm{RG}$ & $\mathrm{F}$ & 75 & 60,00 & 34,63 & 29,48 & 2001 & 2015 & 12 \\
\hline & & $M$ & 50 & 40,00 & 42,54 & 33,00 & 2001 & 2015 & 14 \\
\hline \multirow{4}{*}{ FCE } & SEDICl & $\mathrm{F}$ & 115 & 55,56 & 2,09 & 2,03 & 2004 & 2008 & 4 \\
\hline & & $M$ & 92 & 44,44 & 2,27 & 1,79 & 2004 & 2009 & 26 \\
\hline & $\mathrm{RG}$ & $\mathrm{F}$ & 95 & 56,55 & 32,69 & 26,12 & 1996 & 2015 & 19 \\
\hline & & $\mathrm{M}$ & 73 & 43,45 & 56,55 & 65,92 & 1997 & 2015 & 19 \\
\hline
\end{tabular}

Tabla 3. Presencia y producción de docentes-investigadores en los repositorios y en ResearchGate por género 


\begin{tabular}{|c|c|c|c|c|c|c|c|c|c|c|}
\hline & & \multicolumn{2}{|c|}{ Docinv } & \multicolumn{2}{|c|}{ Documentos } & \multicolumn{2}{|c|}{ Año i } & \multicolumn{2}{|c|}{ Año $f$} & \multirow[b]{2}{*}{$\begin{array}{c}\text { Cobertura } \\
\text { temporal } \\
\text { (Años) }\end{array}$} \\
\hline & $\begin{array}{l}\text { Grupo } \\
\text { etáreo }\end{array}$ & Nro & $\%$ & Media & Desv. Tip. & Media & Desv. Tip. & Media & Desv. Tip. & \\
\hline \multirow{4}{*}{$\begin{array}{c}\text { Memoria } \\
\text { Académica }\end{array}$} & $30-39$ & 4 & 3,74 & 6,50 & 4,93 & 2002 & 1,41 & 2011 & 5,56 & 9 \\
\hline & $40-49$ & 43 & 40,19 & 11,86 & 9,17 & 2003 & 4,86 & 2014 & 1,54 & 11 \\
\hline & $50-59$ & 49 & 45,79 & 13,24 & 9,98 & 2002 & 5,74 & 2014 & 1,63 & 12 \\
\hline & $60 y+$ & 11 & 10,28 & 17,82 & 14,08 & 1997 & 4,60 & 2014 & 1,38 & 17 \\
\hline \multirow{4}{*}{ SEDICI } & $30-39$ & 5 & 4,50 & 10,80 & 5,81 & 2002 & 1,58 & 2013 & 1,64 & 11 \\
\hline & $40-49$ & 44 & 39,64 & 8,16 & 4,76 & 2003 & 5,43 & 2013 & 1,92 & 10 \\
\hline & $50-59$ & 51 & 45,95 & 10,14 & 6,68 & 2000 & 5,57 & 2013 & 1,97 & 13 \\
\hline & $60 y+$ & 11 & 9,91 & 11,27 & 9,82 & 1996 & 8,08 & 2011 & 5,93 & 15 \\
\hline \multirow{4}{*}{ RG } & $30-39$ & 1 & 5,56 & 55,00 & -- & 2009 & -- & 2015 & -- & 6 \\
\hline & $40-49$ & 6 & 33,33 & 20,67 & 18,22 & 2004 & 4,08 & 2013 & 3,52 & 9 \\
\hline & $50-59$ & 10 & 55,56 & 12,30 & 16,26 & 2004 & 5,23 & 2012 & 4,45 & 7 \\
\hline & $60 y+$ & 1 & 5,56 & 14,00 & -- & 2003 & -- & 2010 & -- & 7 \\
\hline
\end{tabular}

Tabla 4.a Presencia y producción de docentes-investigadores en repositorios y en ResearchGate por grupo etáreo- FAHCE

\begin{tabular}{|c|c|c|c|c|c|c|c|c|c|c|}
\hline & & \multicolumn{2}{|c|}{ Docinv } & \multicolumn{2}{|c|}{ Documentos } & \multicolumn{2}{|c|}{ Ano $\mathrm{i}$} & \multicolumn{2}{|c|}{ Ano $f$} & \multirow[b]{2}{*}{$\begin{array}{c}\text { Cobertura } \\
\text { temporal } \\
\text { (Años) }\end{array}$} \\
\hline & $\begin{array}{l}\text { Grupo } \\
\text { etáreo }\end{array}$ & Nro & $\%$ & Media & $\begin{array}{c}\text { Desv. } \\
\text { Tip. }\end{array}$ & Media & $\begin{array}{c}\text { Desv. } \\
\text { Tip. }\end{array}$ & Media & $\begin{array}{c}\text { Desv. } \\
\text { Tip. }\end{array}$ & \\
\hline \multirow{4}{*}{ Naturalis } & $30-39$ & 30 & 15,87 & 18,70 & 11,22 & 2005 & 3,06 & 2014 & 1,01 & 9 \\
\hline & $40-49$ & 62 & 32,80 & 24,18 & 15,67 & 2002 & 3,56 & 2014 & 1,41 & 12 \\
\hline & $50-59$ & 58 & 30,69 & 39,33 & 25,92 & 1996 & 6,13 & 2013 & 1,79 & 17 \\
\hline & $60 y+$ & 39 & 20,63 & 52,21 & 32,50 & 1990 & 7,41 & 2013 & 1,46 & 15 \\
\hline \multirow{4}{*}{ SEDICI } & $30-39$ & 30 & 17,14 & 2,23 & 1,92 & 2009 & 3,15 & 2011 & 2,33 & 2 \\
\hline & $40-49$ & 57 & 32,57 & 5,11 & 6,75 & 2002 & 9,69 & 2009 & 5,04 & 7 \\
\hline & $50-59$ & 54 & 30,86 & 3,94 & 3,79 & 1997 & 8,63 & 2009 & 6,33 & 12 \\
\hline & $60 y+$ & 34 & 19,43 & 6,59 & 9,80 & 1989 & 10,88 & 2007 & 10,40 & 15 \\
\hline \multirow{4}{*}{$\mathrm{RG}$} & $30-39$ & 27 & 21,60 & 25,37 & 12,60 & 2006 & 2,90 & 2015 & 0,52 & 9 \\
\hline & $40-49$ & 46 & 36,80 & 29,30 & 18,77 & 2002 & 3,67 & 2015 & 1,25 & 12 \\
\hline & $50-59$ & 30 & 24,00 & 46,70 & 35,42 & 1998 & 6,86 & 2015 & 1,09 & 17 \\
\hline & $60 y+$ & 22 & 17,60 & 58,64 & 45,67 & 1997 & 10,65 & 2015 & 0,84 & 12 \\
\hline
\end{tabular}

Tabla 4.b Presencia y producción de docentes-investigadores en repositorios y en ResearchGate por grupo etáreo- FCNyM 


\begin{tabular}{|c|c|c|c|c|c|c|c|c|c|c|}
\hline & & \multicolumn{2}{|c|}{ Docinv } & \multicolumn{2}{|c|}{ Documentos } & \multicolumn{2}{|c|}{ Ano $\mathrm{i}$} & \multicolumn{2}{|c|}{ Ano $f$} & \multirow[b]{2}{*}{$\begin{array}{c}\text { Cobertura } \\
\text { temporal } \\
\text { (Años) }\end{array}$} \\
\hline & $\begin{array}{l}\text { Grupo } \\
\text { etáreo }\end{array}$ & Nro & $\%$ & Media & $\begin{array}{c}\text { Desv. } \\
\text { Tip. }\end{array}$ & Media & $\begin{array}{c}\text { Desv. } \\
\text { Tip. }\end{array}$ & Media & $\begin{array}{c}\text { Desv. } \\
\text { Tip. }\end{array}$ & \\
\hline \multirow[b]{4}{*}{ SEDICI } & $30-39$ & 37 & 17,87 & 2,16 & 1,91 & 2009 & 3,38 & 2011 & 2,86 & 2,30 \\
\hline & $40-49$ & 80 & 38,65 & 1,84 & 1,27 & 2006 & 4,08 & 2009 & 4,81 & 3,10 \\
\hline & 50-59 & 70 & 33,82 & 2,34 & 2,29 & 2000 & 9,25 & 2007 & 9,20 & 35,07 \\
\hline & 60 y más & 20 & 9,66 & 2,90 & 2,55 & 2002 & 10,48 & 2008 & 10,00 & 3,81 \\
\hline \multirow[b]{4}{*}{ RG } & $30-39$ & 22 & 13,10 & 17,55 & 10,76 & 2006 & 2,74 & 2015 & 1,27 & 8,77 \\
\hline & $40-49$ & 70 & 41,67 & 25,20 & 16,93 & 2000 & 4,75 & 2015 & 1,16 & 14,79 \\
\hline & 50-59 & 58 & 34,52 & 54,86 & 33,95 & 1991 & 7,11 & 2015 & 1,26 & 24,17 \\
\hline & 60 y más & 18 & 10,71 & 105,67 & 107,73 & 1985 & 9,82 & 2015 & 0,70 & 30,83 \\
\hline
\end{tabular}

Tabla 4.c Presencia y producción de docentes-investigadores en repositorios y en ResearchGate por grupo etáreo- FCE

\begin{tabular}{|c|c|c|c|c|c|c|c|c|c|c|}
\hline & & \multicolumn{2}{|c|}{ Docinv } & \multicolumn{2}{|c|}{ Documentos } & \multicolumn{2}{|c|}{ Año i } & \multicolumn{2}{|c|}{ Año $f$} & \multirow[b]{2}{*}{$\begin{array}{c}\text { Cobertura } \\
\text { temporal } \\
\text { (Años) }\end{array}$} \\
\hline & $\begin{array}{l}\text { Formación } \\
\text { académica }\end{array}$ & Nro & $\%$ & Media & $\begin{array}{c}\text { Desv. } \\
\text { Tip. }\end{array}$ & Media & $\begin{array}{c}\text { Desv. } \\
\text { Tip. }\end{array}$ & Media & $\begin{array}{c}\text { Desv. } \\
\text { Tip. }\end{array}$ & \\
\hline \multirow{6}{*}{$\begin{array}{c}\text { Memoria } \\
\text { Académica }\end{array}$} & Postdoctorado & 17 & 15,89 & 15,12 & 8,54 & 2001 & 5,04 & 2014 & 0,86 & 13,7 \\
\hline & Doctorado & 57 & 53,27 & 13,91 & 10,02 & 2001 & 5,44 & 2014 & 1,96 & 12,6 \\
\hline & Maestría & 16 & 14,95 & 8,38 & 6,55 & 2004 & 3,95 & 2014 & 2,10 & 9,4 \\
\hline & Especialización & 2 & 1,87 & 7,00 & 4,24 & 2001 & 6,36 & 2013 & 0,71 & 12,0 \\
\hline & Grado & 12 & 11,21 & 13,50 & 15,65 & 2003 & 6,45 & 2013 & 2,21 & 10,3 \\
\hline & Sin datos & 3 & 2,80 & 7,00 & 5,20 & 2002 & 8,74 & 2013 & 1,53 & 11,0 \\
\hline \multirow[b]{6}{*}{ SEDICI } & Postdoctorado & 19 & 17,12 & 11,68 & 8,21 & 2000 & 5,36 & 2014 & 1,57 & 13,6 \\
\hline & Doctorado & 59 & 53,15 & 10,14 & 6,15 & 2000 & 5,93 & 2013 & 3,23 & 12,9 \\
\hline & Maestría & 16 & 14,41 & 5,88 & 2,85 & 2004 & 5,75 & 2014 & 1,09 & 9,3 \\
\hline & Especialización & 2 & 1,80 & 7,00 & 4,24 & 2001 & 6,36 & 2014 & 2,12 & 13,0 \\
\hline & Grado & 12 & 10,81 & 8,83 & 6,48 & 2002 & 6,75 & 2013 & 2,61 & 11,1 \\
\hline & Sin datos & 3 & 2,70 & 6,67 & 6,03 & 2002 & 8,19 & 2013 & 1,53 & 10,7 \\
\hline \multirow[b]{4}{*}{ RG } & Postdoctorado & 4 & 22,22 & 16,50 & 3,00 & 2004 & 4,36 & 2015 & 0,50 & 8,0 \\
\hline & Doctorado & 11 & 61,11 & 21,73 & 22,29 & 2005 & 5,29 & 2012 & 3,50 & 7,5 \\
\hline & Maestría & 2 & 11,11 & 2,00 & 1,41 & 2005 & 2,12 & 2006 & 3,54 & 11,3 \\
\hline & Sin datos & 1 & 5,56 & 7,00 & -- & 2007 & -- & 2015 & -- & 1,0 \\
\hline
\end{tabular}




\begin{tabular}{|c|c|c|c|c|c|c|c|c|c|c|}
\hline & & \multicolumn{2}{|c|}{ Docinv } & \multicolumn{2}{|c|}{ Documentos } & \multicolumn{2}{|c|}{ Año i } & \multicolumn{2}{|c|}{ Año $f$} & \multirow[b]{2}{*}{$\begin{array}{c}\text { Cobertura } \\
\text { temporal } \\
\text { (Años) }\end{array}$} \\
\hline & $\begin{array}{l}\text { Formación } \\
\text { académica }\end{array}$ & Nro & $\%$ & Media & $\begin{array}{l}\text { Desv. } \\
\text { Tip. }\end{array}$ & Media & $\begin{array}{l}\text { Desv. } \\
\text { Tip. }\end{array}$ & Media & $\begin{array}{l}\text { Desv. } \\
\text { Tip. }\end{array}$ & \\
\hline \multirow{6}{*}{ Naturalis } & Postdoctorado & 42 & 22,11 & 31,26 & 18,00 & 2001 & 5,35 & 2014 & 1,14 & 11,9 \\
\hline & Doctorado & 122 & 64,21 & 36,61 & 28,96 & 1997 & 7,94 & 2014 & 1,36 & 15,2 \\
\hline & Maestría & 4 & 2,11 & 7,25 & 5,12 & 2003 & 3,70 & 2011 & 4,55 & 8,5 \\
\hline & Especialización & 3 & 1,58 & 8,67 & 6,51 & 2000 & 4,04 & 2010 & 4,62 & 10,7 \\
\hline & Grado & 19 & 10,00 & 22,68 & 16,10 & 1999 & 8,21 & 2013 & 1,50 & 12,5 \\
\hline & Sin datos & 4 & 2,11 & 35,50 & 14,89 & 1999 & 2,38 & 2013 & 2,06 & 14,3 \\
\hline \multirow{6}{*}{ SEDICI } & Postdoctorado & 42 & 24,00 & 3,57 & 3,78 & 2001 & 10,27 & 2008 & 7,26 & 8,24 \\
\hline & Doctorado & 113 & 64,57 & 4,68 & 6,87 & 1999 & 10,91 & 2009 & 6,66 & 10,58 \\
\hline & Maestría & 3 & 1,71 & 3,00 & 1,00 & 2001 & 6,55 & 2012 & 2,51 & 11,67 \\
\hline & Especialización & 3 & 1,71 & 1,00 & 0,00 & 2011 & 4,16 & 2011 & 4,16 & 0,00 \\
\hline & Grado & 12 & 6,86 & 6,58 & 6,64 & 2000 & 9,74 & 2011 & 3,82 & 11,20 \\
\hline & Sin datos & 5 & 2,86 & 6,80 & 10,31 & 2000 & 14,65 & 2013 & 2,88 & $12,8 \mathrm{o}$ \\
\hline \multirow{6}{*}{$R G$} & Postdoctorado & 33 & 26,40 & 38,55 & 23,09 & 2002 & 5,88 & 2015 & 0,77 & 11,12 \\
\hline & Doctorado & 81 & 64,80 & 39,52 & 34,76 & 2000 & 7,56 & 2015 & 0,99 & 13,27 \\
\hline & Maestría & 2 & -- & 12,00 & 15,50 & 2008 & 4,24 & 2013 & 2,82 & 5,00 \\
\hline & Especialización & 1 & 0,80 & 15,00 & -- & 1995 & -- & 2015 & -- & 20,00 \\
\hline & Grado & 7 & 5,60 & 20,71 & 16,68 & 2001 & 6,63 & 2014 & 1,86 & 10,89 \\
\hline & Sin datos & 3 & 2,40 & 30,33 & 24,58 & 2008 & 2,89 & 2014 & 0,58 & 6,00 \\
\hline
\end{tabular}

Tabla 5.b Presencia y producción de docentes-investigadores en repositorios y en ResearchGate por formación académica- FCNyM

\begin{tabular}{|c|c|c|c|c|c|c|c|c|c|c|}
\hline & \multirow[b]{2}{*}{$\begin{array}{l}\text { Formación } \\
\text { académica }\end{array}$} & \multicolumn{2}{|c|}{ Docinv } & \multicolumn{2}{|c|}{ Documentos } & \multicolumn{2}{|c|}{ Año i } & \multicolumn{2}{|c|}{ Año $f$} & \multirow[b]{2}{*}{$\begin{array}{c}\text { Cobertura } \\
\text { temporal } \\
\text { (Años) }\end{array}$} \\
\hline & & Nro & $\%$ & Media & $\begin{array}{c}\text { Desv. } \\
\text { Tip. }\end{array}$ & Media & $\begin{array}{c}\text { Desv. } \\
\text { Tip. }\end{array}$ & Media & $\begin{array}{c}\text { Desv. } \\
\text { Tip. }\end{array}$ & \\
\hline \multirow{6}{*}{ SEDICI } & Postdoctorado & 113 & 54,59 & 2,22 & 1,90 & 2004 & 7,36 & 2009 & 6,79 & 4,05 \\
\hline & Doctorado & 83 & 40,10 & 2,18 & 2,06 & 2004 & 7,58 & 2009 & 7,14 & 27,45 \\
\hline & Maestría & 1 & 0,48 & 1,00 & -- & 2001 & -- & 2001 & -- & 0,00 \\
\hline & Especialización & 2 & 0,97 & 1,00 & 0,00 & 2013 & 0,71 & 2013 & 0,71 & 0,00 \\
\hline & Grado & 5 & 2,42 & 1,60 & 0,89 & 2012 & 2,95 & 2013 & 3,39 & 0,67 \\
\hline & Sin datos & 4 & 1,93 & 1,75 & 0,96 & 1996 & 10,17 & 1999 & 12,52 & 3,00 \\
\hline \multirow{5}{*}{$\mathrm{RG}$} & Postdoctorado & 102 & 60,71 & 40,96 & 29,83 & 1997 & 8,60 & 2015 & 0,94 & 18,80 \\
\hline & Doctorado & 61 & 36,31 & 49,13 & 71,06 & 1995 & 9,85 & 2015 & 1,41 & 19,60 \\
\hline & Maestría & -- & -- & -- & -- & -- & -- & -- & -- & -- \\
\hline & Especialización & 2 & 1,19 & 7,50 & 7,78 & 2006 & 7,07 & 2015 & 0,71 & -- \\
\hline & Grado & 3 & 1,79 & 14,67 & 0,58 & 1998 & 5,51 & 2013 & 0,58 & -- \\
\hline
\end{tabular}




\begin{tabular}{|c|c|c|c|c|c|c|c|c|c|c|}
\hline & \multirow[b]{2}{*}{ Categoría } & \multicolumn{2}{|c|}{ Docinv } & \multicolumn{2}{|c|}{ Documentos } & \multicolumn{2}{|c|}{ Año i } & \multicolumn{2}{|c|}{ Año $f$} & \multirow[b]{2}{*}{$\begin{array}{c}\text { Cobertura } \\
\text { temporal } \\
\text { (Años) }\end{array}$} \\
\hline & & Nro & $\%$ & Media & $\begin{array}{c}\text { Desv. } \\
\text { Tip. }\end{array}$ & Media & $\begin{array}{c}\text { Desv. } \\
\text { Tip. }\end{array}$ & Media & $\begin{array}{c}\text { Desv. } \\
\text { Tip. }\end{array}$ & \\
\hline \multirow{5}{*}{$\begin{array}{c}\text { Memoria } \\
\text { Académica }\end{array}$} & $\mathrm{I}$ & 9 & 8,41 & 16,33 & 8,99 & 2001 & 6,51 & 2014 & 1,32 & 12,9 \\
\hline & II & 24 & 22,43 & 15,92 & 12,12 & 2001 & 5,03 & 2014 & 1,25 & 13,4 \\
\hline & III & 48 & 44,86 & 14,06 & 10,31 & 2001 & 5,18 & 2014 & 1,07 & 13,2 \\
\hline & IV & 17 & 15,89 & 7,35 & 4,49 & 2003 & 5,17 & 2012 & 3,16 & 9,3 \\
\hline & $\mathrm{V}$ & 9 & 8,41 & 5,78 & 4,06 & 2007 & 4,59 & 2013 & 2,45 & 6,1 \\
\hline \multirow{5}{*}{ SEDICI } & 1 & 9 & 8,11 & 12,11 & 7,29 & 1999 & 5,81 & 2013 & 1,83 & 13,6 \\
\hline & II & 25 & 22,52 & 10,88 & 7,74 & 1999 & 5,65 & 2012 & 4,26 & 12,8 \\
\hline & III & 50 & 45,05 & 9,98 & 6,11 & 2000 & 6,01 & 2014 & 1,84 & 13,8 \\
\hline & IV & 18 & 16,22 & 5,89 & 3,23 & 2004 & 5,64 & 2013 & 1,50 & 9,5 \\
\hline & V & 9 & 8,11 & 7,56 & 4,85 & 2006 & 4,12 & 2013 & 3,03 & 6,4 \\
\hline \multirow{5}{*}{ RG } & I & 3 & 16,67 & 12,00 & 3,46 & 2007 & 3,51 & 2013 & 2,65 & 6,3 \\
\hline & II & 2 & 11,11 & 35,00 & 29,70 & 1997 & 7,07 & 2015 & 0,00 & 18,0 \\
\hline & III & 12 & 66,67 & 17,25 & 18,99 & 2005 & 3,61 & 2012 & 4,36 & 6,8 \\
\hline & IV & 1 & 5,56 & 3,00 & & 2006 & & 2008 & & 2,0 \\
\hline & $\mathrm{V}$ & -- & -- & -- & -- & -- & -- & -- & -- & -- \\
\hline
\end{tabular}

Tabla 6.a. Presencia y producción de docentes-investigadores en repositorios y en ResearchGate por categoría de investigador - FAHCE

\begin{tabular}{|c|c|c|c|c|c|c|c|c|c|c|}
\hline & & \multicolumn{2}{|c|}{ Docinv } & \multicolumn{2}{|c|}{ Documentos } & \multicolumn{2}{|c|}{ Año i } & \multicolumn{2}{|c|}{ Año $f$} & \multirow[b]{2}{*}{$\begin{array}{c}\text { Cobertura } \\
\text { temporal } \\
\text { (Años) }\end{array}$} \\
\hline & Categoría & Nro & $\%$ & Media & $\begin{array}{c}\text { Desv. } \\
\text { Tip. }\end{array}$ & Media & $\begin{array}{c}\text { Desv. } \\
\text { Tip. }\end{array}$ & Media & $\begin{array}{c}\text { Desv. } \\
\text { Tip. }\end{array}$ & \\
\hline \multirow{5}{*}{ Naturalis } & 1 & 29 & 15,26 & 69,62 & 32,16 & 1989 & 6,53 & 2014 & 1,21 & 24,6 \\
\hline & II & 30 & 15,79 & 42,67 & 17,16 & 1994 & 7,20 & 2014 & 1,61 & 19,3 \\
\hline & III & 63 & 33,16 & 31,94 & 18,07 & 1999 & 5,54 & 2013 & 1,42 & 14,3 \\
\hline & IV & 43 & 22,63 & 17,91 & 9,84 & 2002 & 4,04 & 2014 & 1,32 & 11,3 \\
\hline & $\mathrm{V}$ & 25 & 13,16 & 11,92 & 7,14 & 2005 & 3,82 & 2014 & 2,20 & 5,4 \\
\hline \multirow{5}{*}{ SEDICI } & 1 & 28 & 16,00 & 7,54 & 10,75 & 1988 & 9,84 & 2006 & 11,29 & 18,6 \\
\hline & II & 28 & 16,00 & 3,46 & 2,52 & 1994 & 7,73 & 2008 & 6,79 & 14,8 \\
\hline & III & 57 & 32,57 & 5,81 & 6,88 & 2000 & 10,53 & 2009 & 6,03 & 9,6 \\
\hline & IV & 37 & 21,14 & 2,51 & 1,91 & 2006 & 3,64 & 2010 & 2,97 & 7,7 \\
\hline & $\mathrm{V}$ & 25 & 14,29 & 2,52 & 3,12 & 2008 & 5,48 & 2011 & 2,56 & 2,7 \\
\hline \multirow{5}{*}{ RG } & 1 & 19 & 15,20 & 78,63 & 46,03 & 1995 & 10,70 & 2015 & 0,78 & 20,4 \\
\hline & II & 17 & 13,60 & 48,94 & 30,07 & 1997 & 6,61 & 2015 & 1,01 & 17,6 \\
\hline & III & 36 & 28,80 & 35,11 & 18,12 & 2001 & 4,79 & 2015 & 1,26 & 13,9 \\
\hline & IV & 35 & 28,00 & 21,60 & 13,21 & 2004 & 3,84 & 2015 & 1,01 & 10,3 \\
\hline & V & 18 & 14,4 & 21 & 8,51 & 2006,56 & 3,17 & 2014,89 & 0,68 & 4,84 \\
\hline
\end{tabular}




\begin{tabular}{|c|c|c|c|c|c|c|c|c|c|c|}
\hline & \multirow[b]{2}{*}{ Categoría } & \multicolumn{2}{|c|}{ Docinv } & \multicolumn{2}{|c|}{ Documentos } & \multicolumn{2}{|c|}{ Año i } & \multicolumn{2}{|c|}{ Año $f$} & \multirow[b]{2}{*}{$\begin{array}{c}\text { Cobertura } \\
\text { temporal } \\
\text { (Años) }\end{array}$} \\
\hline & & Nro & $\%$ & Media & $\begin{array}{c}\text { Desv. } \\
\text { Tip. }\end{array}$ & Media & $\begin{array}{c}\text { Desv. } \\
\text { Tip. }\end{array}$ & Media & $\begin{array}{c}\text { Desv. } \\
\text { Tip. }\end{array}$ & \\
\hline \multirow{5}{*}{ SEDICI } & 1 & 26 & 12,56 & 3,04 & 2,55 & 2000 & 10,06 & 2010 & 7,58 & 6,9 \\
\hline & II & 39 & 18,84 & 2,31 & 1,95 & 2000 & 9,65 & 2005 & 10,67 & 5,1 \\
\hline & III & 64 & 30,92 & 2,13 & 1,99 & 2003 & 5,73 & 2008 & 6,49 & 35,9 \\
\hline & IV & 49 & 23,67 & 1,86 & 1,44 & 2007 & 4,08 & 2010 & 4,46 & 2,1 \\
\hline & $\mathrm{V}$ & 29 & 14,01 & 1,83 & 1,69 & 2010 & 2,37 & 2012 & 2,04 & 1,4 \\
\hline \multirow{5}{*}{$\mathrm{RG}$} & 1 & 28 & 16,67 & 99,82 & 86,68 & 1987 & 8,75 & 2015 & 0,73 & 28,6 \\
\hline & II & 36 & 21,43 & 56,61 & 27,94 & 1991 & 5,44 & 2015 & 0,70 & 24,2 \\
\hline & III & 46 & 27,38 & 31,61 & 21,71 & 1996 & 8,08 & 2015 & 1,40 & 19,0 \\
\hline & IV & 40 & 23,81 & 17,70 & 10,21 & 2003 & 3,68 & 2015 & 1,39 & 12,1 \\
\hline & $\mathrm{V}$ & 18 & 10,71 & 13,28 & 5,30 & 2006 & 2,91 & 2015 & 1,14 & 8,6 \\
\hline
\end{tabular}

Tabla 6. c. Presencia y producción de docentes-investigadores en repositorios y en ResearchGate por categoría de investigador - FCE 
